Mots. Les langages du politique

Présidentielle 2007. Scènes de genre

Ségolène Royal ou le difficile accès au panthéon politique

Isabelle Garcin-Marrou

\title{
OpenEdition
}

Journals

Édition électronique

URL : https://journals.openedition.org/mots/19074

DOI : $10.4000 /$ mots. 19074

ISSN : 1960-6001

Éditeur

ENS Éditions

Édition imprimée

Date de publication : 1 juillet 2009

Pagination : 13-29

ISBN : 978-2-84788-151-6

ISSN : 0243-6450

Référence électronique

Isabelle Garcin-Marrou, "Ségolène Royal ou le difficile accès au panthéon politique », Mots. Les

langages du politique [En ligne], 90 | 2009, mis en ligne le 01 juillet 2011, consulté le 22 avril 2022. URL:

http://journals.openedition.org/mots/19074; DOI : https://doi.org/10.4000/mots.19074 


\section{Ségolène Royal ou le difficile accès au panthéon politique}

La campagne présidentielle de 2007, qui a vu Ségolène Royal porter la candidature du Parti socialiste face à Nicolas Sarkozy au second tour de l'élection, semble avoir été implicitement irriguée, dans les discours tant politiques que médiatiques, par la question cruciale, quoique restée implicite: une femme politique, est-ce un personnage politique désigné grammaticalement par son genre ou un personnage politique dont la caractéristique essentielle serait le féminin ? Les réponses à cette question peuvent permettre de comprendre comment les représentations médiatiques de la candidate se sont imposées dans l'espace public et ont pu peser sur la trajectoire électorale de Ségolène Royal.

La lecture des très nombreux articles consacrés à la candidate et à ses concurrents a fait apparaitre un certain nombre de qualifications narratives qui interrogeaient vivement le critère du genre en politique. De nombreuses recherches ont déjà montré la puissance de ce critère dans les difficultés des femmes pour être légitimées, notamment par les représentations médiatiques, dans leur accession au pouvoir ou dans son exercice (Achin, Dorlin, 2007, p. $2^{1}$ ). «Le constat d'un renvoi systématique des femmes à leur identité sexuée traditionnelle demeure patent», écrit ainsi Cécile Sourd en conclusion d'une étude menée sur la représentation des candidates aux élections présidentielle et législatives de 2002 (Sourd, 2005, p. 77). II n'est pas ici question de revenir sur des conclusions déjà établies, mais il peut être intéressant de reprendre ces questions pour tenter de saisir quelques éléments d'ancrage anthropologique des représentations médiatiques proposées en 2007.

\footnotetext{
Université Lyon 2, isabelle.garcin-marrou@univ-lyon2.fr
}

1. "On perçoit ici l'effet pervers de la "quadrature du cercle": appelées en politique au nom de leur "différence” et de capitaux politiques spécifiques, historiquement associés à leur sexe (appartenance à la société civile, militantisme associatif, etc.), les femmes sont pénalisées dans la conquête de réelles positions de pouvoir, conquête qui reste attachée à la possession de ressources plus classiques, comme les capitaux partisans ou l'expérience politique. Force est ici de constater l'importante inertie des normes d'excellence politique que la parité ne semble guère avoir bousculées.» 
La contribution de Nicole Loraux à l'Histoire des femmes en Occident, dirigée par Georges Duby et Michelle Perrot (Loraux, 1991), contribution consacrée aux déesses dans le monde grec antique, a permis l'identification de la question initiale et la proposition d'analyse qui en découle. S'appuyant sur l'analyse des dénominations grecques, Nicole Loraux établit l'alternative suivante: soit une déesse n'est que «grammaticalement le féminin du mot Dieu (Theós / Theá)» (Loraux, 1991, p. 42) - Theá étant la forme féminine qui ne permet pas, pour autant, de dire que dans une déesse le féminin l'emporte sur le divin; soit, dans une déesse, le féminin est une caractéristique essentielle, que l'on retrouve exprimée par la maternité et par un éternel féminin qui permet de replacer la Mère à l'origine, de lui assigner un pouvoir au début de l'histoire, pour lui dénier ensuite la prétention d'avoir ce pouvoir: "Jadis fonde maintenant. » (ibid., p. 70)

Les déesses du panthéon grec, en qui prime le divin, sont représentées comme dotées d'une qualité première, la beauté. "La beauté divine est par essence "pure", et superlative en ce qu'elle exprime l'être-dieu», la beauté des déesses du panthéon étant par ailleurs «l'expression nécessaire de leur puissance» (Loraux, 1991, p. 49). À ces déesses du panthéon sont interdites la proximité avec les mortels ou l'expression d'une émotion. La scène de la tragédie d’Euripide citée par Nicole Loraux, Hippolyte porte-couronnes, décrit ainsi la déesse Artémis s’interdisant d'approcher Hippolyte, son protégé, et de pleurer sa mort prochaine. Enfin, «si la beauté est aux déesses, aux femmes appartient la voix, en tant qu'elles sont mortelles » (ibid., p. 50). Une déesse du panthéon n'est jamais qualifiée par sa voix et lorsqu'il y a mention de cette qualité, le texte grec «juxtapose en un superbe oxymoron l'être-dieu, la voix humaine et le féminin [...] en une contiguïté dont le désaccord entre les genres (une terminaison féminine, deiné / une de forme masculine, theos / un féminin, audessa) suggère qu'elle dissimule de l'inconciliable» (ibid., p. 50).

Cette césure symbolique entre les déesses du panthéon et les autres déesses, au sujet desquelles Nicole Loraux rappelle qu'«il y a des déesses mais que le divin ne s'énonce pas au féminin » (ibid., p. 43), constitue, par analogie, un angle heuristique pour l'analyse des discours médiatiques de la campagne présidentielle. L'être-femme de Ségolène Royal a constitué un incontestable élément de la campagne comme des commentaires de la campagne. Or le rappel de cet être-femme doit aussi être envisagé comme un élément possible de la disqualification politique de la candidate dans les représentations collectives. Nous pouvons faire l'hypothèse que cette campagne et ses représentations médiatiques, en insistant sur la qualification de Ségolène Royal comme femme, ont pu éloigner symboliquement la figure de la candidate d'un possible accès à la place politique suprême, d'un accès, donc, au panthéon politique. Il ne nous revient pas de juger de la pertinence de la campagne menée 
par Ségolène Royal, mais cette réassignation de la candidate à son genre, coconstruite par elle-même ${ }^{2}$, a ailleurs été analysée, comme un «piège » se refermant sur elle (de Singly, 20083).

Des éditions de presse quotidienne nationale et de presse magazine, parues à des moments clés de la campagne de 2007 , sont mobilisées pour tenter de comprendre ce qui prime (le politique ou le féminin) dans les discours. Quand les journaux parlent de Ségolène Royal, parlent-ils d'un candidatfemme ou d'une femme-candidate? Les vingt-sept articles retenus sont consacrés aux deux candidats et le corpus réunit trois séries d'articles. Première série: Marianne, L'Express et Le Monde ont proposé soit un dossier consacré de façon comparative aux deux candidats, soit des reportages-portraits consacrés à chacun des deux candidats et publiés dans des éditions successives. Deuxième et troisième séries: des journaux nationaux - L'Humanité, Libération, Le Monde, Aujourd'hui en France et Le Figaro - parus le lendemain du discours d'investiture de Nicolas Sarkozy à Versailles le 15 janvier 2007 et le lendemain du «meeting d’intronisation» (Coulomb-Gully, 2009, p. 31) de Ségolène Royal à Villepinte le 11 février $2007^{4}$.

S’il ne peut rendre compte de l'intégralité des discours tenus sur les deux candidats, l'ensemble de ces articles offre une dimension comparative interne qui permet l'analyse. Les discours médiatiques ont été produits en des circonstances politiques similaires ou dans des dispositifs médiatiques symétriques; l'analyse peut donc se centrer sur les variations que ni le type de discours, ni les circonstances politiques ne peuvent expliquer de façon première. Les catégories issues de la réflexion de Nicole Loraux guident l'analyse et permettent de comprendre comment les mentions de la qualité de femme peuvent éloigner Ségolène Royal du panthéon politique légitime en cette campagne présidentielle, comment se forgent, donc, des représentations médiatiques, anthropologiquement structurées, de ce que serait le politique. La comparaison avec les discours tenus sur Nicolas Sarkozy permet de vérifier si les mentions du genre sont liées aux circonstances médiatiques et politiques ou si elles sont une spécificité des discours médiatiques tenus sur Ségolène Royal.

Les discours de la presse sont ici analysés à partir de leur organisation narrative (Courtès, 1991). Les éléments discursifs d'institution et de qualification

2. C'est notamment la déclaration abondamment commentée, "Je sais, en tant que mère ", lors du discours d'«intronisation de la candidate», le 11 février 2007 à Villepinte. Voir CoulombGully, 2009, p. 31 et 36.

3. «Clairement, aujourd'hui, dans nos têtes, on ne confie pas la présidence de la République à un individu considéré comme proche (signe du prénom), on le confie à quelqu'un de compétent, à quelqu'un qu'on n'ose désigner que par son nom. » (de Singly, 2007) Le titre d'un article de Luc Le Vaillant, «Pour en finir avec Calamity Sego », paru dans Libération au lendemain du second tour de l'élection, le 8 mai 2007, illustre la mécanique symbolique du piège alors refermé.

4. Pour la présentation détaillée et les références des articles analysés, se reporter à la fin de l'article. 
des sujets - les candidats - sont lus à partir des catégories dont Nicole Loraux montre le caractère opératoire pour déterminer ce qui, du féminin ou du divin, prime dans la déesse : la maternité, la beauté, la voix, l'émotion et la proximité5. L'organisation narrative des discours implique la sanction narrative des sujets qu'ils instaurent et font agir; les éléments d'institution et de qualification présents dans les discours participent de la sanction, positive ou négative, des sujets dans et par le récit. La sanction narrative n'intéresse pas notre propos en tant que telle, mais parce qu'elle est susceptible de produire la réassignation au féminin. Comme le remarquent Catherine Achin et Elsa Dorlin, Ségolène Royal «a été à de nombreuses reprises mise en question et ses "bourdes" particulièrement commentées». Elle a eu, alors, un "réflexe de protection» qui l'a amenée à se replonger «dans les habits de la "femme politique" oxymorique» (Achin et Dorlin, 2007, p. 23). Les discours sont donc analysés à partir de la présence d'éléments susceptibles de (co-)construire cette réassignation au féminin ; l'analyse des discours tenus sur Nicolas Sarkozy permet de vérifier si l'assignation au genre est partagée ou spécifique à la candidate.

\section{La Mère, la femme}

L'expérience de la maternité a été mise en valeur par la candidate elle-même lors de son discours à Villepinte. Cette (auto)définition par la maternité a fait l'objet de commentaires médiatiques très nombreux, qui ont souligné plutôt positivement la vérité et l'émotion sincère exprimée par Ségolène Royal6. Ce faisant, les discours ont validé le recours de cette dernière à son expérience de femme:

La candidate qui s'est exclamée [...] pour dire sa conviction: «Je veux réaliser pour chaque enfant né ici ce que j'ai voulu pour mes propres enfants.» (Le Monde, d)

S'éloignant de son discours écrit, Royal, soudain, parle d'elle: «Je sais au fond de moi, en tant que mère, que je veux [...] » (Aujourd'hui en France, e)

Et le poing sur le ventre: «[...] Ce que je veux pour chaque jeune, c'est ce que j'ai voulu comme mère pour mes propres enfants » [...] (Le Figaro, c)

Mutation, enfin, vers une stature présidentielle, quand la candidate se pose en mère de la Nation [...] (Libération, d)

Définie comme Mère, la candidate est ramenée au pouvoir originel décrit par Nicole Loraux, mais ce pouvoir, qui n'est plus, construit la candidate comme une figure où prime le féminin. Une telle (auto)définition interdit la reconnais-

5. Ces catégories correspondent aussi à celles dont Cécile Sourd a montré la pertinence heuristique (Sourd, 2005).

6. Tous les journaux insistent sur ce passage précis du discours de la candidate, et Libération titrera le 26 mars 2007: « Mère Patrie». 
sance de Ségolène Royal comme appartenant au panthéon politique. La sanction narrative positive de Libération quant à la «mutation vers une stature présidentielle [de la candidate posée en] Mère de la Nation » occulte la réassignation au genre féminin et participe du «piège » décrit par François de Singly. Il faut remarquer que, dans la série d'articles symétriques, Le Monde insiste également sur le statut de mère. Pourtant, pour ce quotidien, le recours à la qualification par la fonction maternelle n'est pas un argument politique valide :

«La différence, je crois qu'elle se voit»; Ségolène Royal n’a pas hésité, parfois, à abuser de sa « différence » [...] ou bien à mettre en scène sa qualité de mère comme un critère d'aptitude à gouverner.

La candidate a fait de l'exercice du pouvoir un élément de clivage [...] en prenant soin d'opposer la «brutalité » de son adversaire masculin à son message de réconciliation. (Le Monde, e)

Le discours pointe l'abus du recours au genre et refuse de sanctionner positivement la figure politique ainsi construite. Notons cependant que cette sanction est relativisée, dans l'article même, par l'abondante description de l'apprêt physique de la candidate. Nous y reviendrons.

À ces qualifications de Ségolène Royal comme mère - comme «Mère superlative» (Coulomb-Gully, 2009, p. 35) - s'ajoute une caractéristique importante de la campagne de 2007 : celle induite par le fait que la candidate du PS était aussi la compagne du premier secrétaire du même parti. Il est intéressant de remarquer que, dans le corpus principal, la thématique est extrêmement présente, mobilisant fortement le genre dans la construction de la figure de la candidate :

La difficulté qu'éprouve le $1^{\text {er }}$ secrétaire à se positionner par rapport à sa compagne $[. .$.

Depuis que Monsieur [...] sans prononcer le nom de Madame, les camarades doivent choisir leur camp, comme les enfants d'un divorce conflictuel [...]

Avec papa, pour revendiquer [...] ou avec maman pour défendre[...]

Certes Royal convient avec son homme [...]

Il tient cette fois-ci en cas de succès ou d'échec de sa «Ségo » [...]

Bref, le compagnon n'a tenu compte d'aucune des réserves de sa compagne [...]

François partage avec Ségolène [...] mais c'est peu dire que le triomphe de sa moitié n'a pas provoqué de débordement d'enthousiasme de sa part au soir de l'investiture de sa compagne [...]

Ségolène Royal, heurtée par la «liberté» que s’est offerte François Hollande, a refusé d'apparaître en femme trahie [...]

À l'inverse de François Hollande, qui tient le parti quand sa compagne continue de le contourner [...]. (Marianne)

L'article de Marianne réitère les éléments de qualification qui assignent Ségolène Royal à son statut de «femme de». Certes, le terme «compagnon» est utilisé en regard du terme "compagne», dans une apparente symétrie de 
désignation de la candidate et du premier secrétaire du PS. Mais il faut noter que le pronom possessif n'apparait qu'une fois pour qualifier le compagnon, quand il apparait à plusieurs reprises pour qualifier la candidate comme compagne - voire comme «moitié» - du premier secrétaire. Le seul élément, notable, inversant la perspective de cet article se trouve dans la citation des propos d'Arnaud Montebourg, porte-parole éphémère de la candidate: " "Ségolène n'a qu'un défaut, c'est son compagnon", a déclaré sur Canal+, "pour de rire", Arnaud Montebourg.» Le propos, pas tout à fait assumé par le porte-parole qui le nuance ensuite en l'inscrivant dans le registre de l'humour, semble pourtant dire exactement le danger des représentations collectives - et médiatiques - de la candidate, qui la ramènent à son statut de «compagne» et l'éloignent de son statut de candidate. L'éclair de lucidité dont semble alors faire preuve Arnaud Montebourg ne se reproduira pas et sera commenté - et jugé - comme un dysfonctionnement politique puisqu'il entrainera la suspension du porte-parole.

Dans les articles consacrés à Nicolas Sarkozy, l'assignation au statut de «mari de » ou à celui de père est très dissemblable. Les articles parus au lendemain du discours de Versailles font à peine mention des statuts paternel et conjugal du candidat. Et lorsque le discours mentionne le mari de Cécilia Sarkozy, c'est pour qualifier cette dernière comme un adjuvant narratif au service des ambitions du candidat:

Face à lui, au huitième rang, [...] ce sont Pierre et Jean, ses deux fils, et Cécilia, son épouse. (Aujourd'hui en France, b)

Cécilia Sarkozy, promue réalisatrice du show de son mari [...]. (Le Figaro, a)

Quant aux articles symétriques, s'ils évoquent la situation conjugale, ils taisent complètement la paternité du candidat.

Son candidat d'époux [...]

[Elle est] le pivot psychologique, médiatique, et donc politique de la campagne du candidat de droite; sa présence est consubstantielle à la stabilité du candidat; ce qu'elle veut [...] c'est que le candidat s'ouvre, s'aère. (Marianne)

Nicolas Sarkozy a laissé son épouse Cécilia recomposer en partie ses équipes; il sait que des murmures sur les états d'âme de sa femme circulent; il refuse d'en dire un mot. (Le Monde, $\mathrm{f}$ )

Marianne attribue à Cécilia Sarkozy un rôle clé - psychologique et politique dans le parcours politique du candidat de l'UMP, qualifiant peut-être également la femme du candidat comme un adjuvant dans la quête du pouvoir politique suprême. Mais il faut également noter que l'usage de l'adjectif possessif se fait en intercalant le terme "candidat» entre l'adjectif et le substantif «époux». Le mari de Cécilia Sarkozy est donc bien qualifié à partir de son statut conjugal, mais cette qualification est relativisée, dans son énonciation 
même, par le rappel de la dimension politique de la figure de Nicolas Sarkozy. L'assignation au statut de «mari de» est donc nuancée aussitôt énoncée. En regard, le discours du Monde est on ne peut plus traditionnel et décrit le candidat comme celui à qui appartient, aussi, une épouse. Pas d'ambigüité, donc, dans la qualification de Nicolas Sarkozy: il est avant tout un politique.

Amorcé par Ségolène Royal elle-même, le piège symbolique a fonctionné. La candidate, (auto)définie comme mère, a été réassignée à son genre, tandis que le candidat conservait une figure politique. Cela semble avoir autorisé, par ailleurs, une insistance sur l'expression d'une appartenance de la candidate à son compagnon. A contrario, les rares mentions du statut conjugal ou de la paternité du candidat n'ont pas été mobilisées comme argument de genre.

\section{La beauté ou l'expression de la puissance en politique}

Tout au long de la campagne, les vêtements portés par Ségolène Royal ont été longuement décrits, et nous ne reviendrons pas ici sur le traitement spécifique réservé à cette question des vêtements des femmes politiques7. Nous voulons simplement rappeler que la description de l'apparence vestimentaire a été particulièrement commentée lors du discours de Ségolène Royal à Villepinte :

Soucieuse de son image, Ségolène Royal avait, hier, abandonné son habituelle tenue blanche pour un tailleur rouge [...]

La candidate, vêtue d'un tailleur rouge [...] (L'Humanité, b)

Ségolène Royal, ensemble rouge vif [...] (Libération, d)

Lorsque Ségolène Royal - vêtue cette fois de rouge et non plus de blanc s'avance [...]

Veste rouge à boutons noirs sur tee-shirt blanc et veste en mousseline orangée, Ségolène Royal fait son entrée. (Aujourd'hui en France, d)

Seule sur une tribune intégralement blanche, Ségolène Royal, vêtue de rouge [...] Ségolène Royal était en rouge [...] (Le Figaro, d)

Or, rien de comparable n'apparait dans les articles consacrés au discours d'investiture de Nicolas Sarkozy. Aucune mention de sa tenue vestimentaire ne peut être relevée. L'asymétrie des discours est là remarquable et participe d'une qualification par le genre spécifique à la candidate. Mais c'est surtout la transformation que décrivent les articles symétriques qui doit être remarquée; transformation liée aux efforts de Ségolène Royal pour apparaitre en phase avec son projet politique :

7. Voir Sourd, 2005, p. 67-70. 
Elle a changé physiquement. "Elle s'est épanouie, souligne un proche. Elle a su mettre sa beauté en valeur, en s'habillant autrement, en femme plus femme.» (Marianne)

Avant, le charme, la «Royal touch » célébrée par la presse anglo-saxonne, aplanissait les difficultés; désormais seule compte la démonstration répétée de l'aptitude au pouvoir. Il est vrai que le propre du charme est de se dissiper. (L'Express, b)

Le diable se niche dans les détails. Au fil des mois, les vêtements portés par la candidate ont sensiblement évolué. À la phase "proche des gens» [...] ont correspondu des tenues simples, féminines - jamais de pantalon -, toujours adaptées. Par exemple, elle portait une veste de cuir noir sur une chemise blanche. Dans une deuxième phase, à un moment où les enquêtes d'opinion soulignaient une carence en crédibilité, Mme Royal a changé de style : tailleur sobre, noir et blanc. Présidentielle. Le costume élyséen est apparu avec la présentation de son programme sur la défense. (Le Monde, e)

Sanction positive des discours, la candidate est décrite comme adaptant ses vêtements aux phases de la campagne. Cette sanction indique cependant, en creux, qu'elle n'est pas prête, d'emblée, pour la fonction à laquelle elle aspire. Libération avait ainsi précisé, après le discours de Villepinte, entretenant l'ambiguïté entre le féminin et le politique: «Ségolène Royal conclut l'exposition de son dessein présidentiel et finit d'ajuster son tailleur de femme d'État.»

Un autre extrait issu du corpus peut retenir l'attention, et ce d'autant plus qu'il s'agit d'une accroche du Monde, particulièrement visible et lisible, donc, dans le dispositif du média ; «On entend le bruit, très bref, d’un sèche-cheveux. Le coiffeur s'éclipse. Pimpante, Ségolène Royal [...] descend à Paris. » Cet incipit est intéressant, car il indique de façon anecdotique que la beauté de Ségolène Royal est construite, que Ségolène Royal s'apprête ${ }^{8}$. L'apprêt physique qu'elle déploie peut donc être compris comme un élément qui la renvoie à sa condition essentiellement féminine - loin du panthéon et loin d'une expression nécessaire de sa puissance politique. En étant décrite comme assumant sa féminité en politique et comme travaillant à la mettre en valeur, la figure de Ségolène Royal apparait comme ne possédant pas la beauté des déesses décrite par Nicole Loraux; beauté «d'une “pureté" quasi chimique» (Loraux, 1991, p. 47). Qualifiée positivement pour sa mutation vestimentaire et corporelle, la candidate est éloignée de celles en qui le divin prime le féminin, en qui, donc, le politique prime le féminin. Cette assignation de Ségolène Royal à son genre par la description vestimentaire peut d'ailleurs être éclairée par le contre-exemple de Martine Aubry qu'analyse Cécile Sourd. Martine Aubry a été qualifiée comme une «femme politique “masculine” »(Sourd, 2005, p. 74), l'expression de la qualité politique n'étant alors pensable qu'en occultant le féminin.

8. Marlène Coulomb-Gully précise que «Ségolène Royal a choisi, pour cette campagne, de se conformer aux critères de la beauté valorisée par la norme contemporaine» (Coulomb-Gully, 2009, p. 28). 
Il faut préciser, pour clore cette question de l'apprêt, que rien n'est dit dans les articles retenus sur un apprêt vestimentaire ou physique de Nicolas Sarkozy. La seule mention explicite est donnée par Le Figaro, qui précise que, avant son discours, «Nicolas Sarkozy [apparait] en costume sombre, chemise blanche, sans cravate ». La description d'une apparence conforme au politique - ou expression même du politique - ne fait donc quasiment pas l'objet d'un discours médiatique, et ce même dans les articles dont l'apparente symétrie masque une forte inégalité de traitement. Cette inégalité se confirme dans les éléments de discours consacrés à la voix des candidats.

\title{
La voix d'une femme
}

Ségolène Royal est aussi décrite à partir de la qualité de sa voix, dont Nicole Loraux rappelle qu'elle est une qualité fondamentalement humaine :

\begin{abstract}
Ségolène Royal a trouvé sa voix. Elle avait jusque-là du mal à la poser [...] Hier le ton était juste, capable d'emporter une salle. (Libération, e)

La voix un peu blanche, la voix, tremblante, s'envole dans les aigus. (Aujourd'hui en France, e)
\end{abstract}

Sa voix s'étrangle. (Le Figaro, e)

Sa voix ne porte pas? Elle impose l'attention. (Le Monde, e)

Décrire la voix de Ségolène Royal, c'est donc évoquer la femme, pas la candidate, même lorsque le discours qualifie positivement la voix et le ton trouvés par elle pour parler en meeting. Comment comprendre, alors, la description faite de la voix de Nicolas Sarkozy?

Sa voix est descendue d'une octave; Nicolas Sarkozy ne parle plus: il murmure. Sa voix de stentor, il ne la réserve plus qu'à ses meetings, ses harangues. (Le Monde, f)

La voix de Nicolas Sarkozy n'est pas tout à fait celle d'un homme; elle est celle de Stentor, guerrier dont l'lliade raconte que la voix exceptionnelle fut utilisée par la déesse Héra pour encouragerl'armée grecque lors du siège de Troie. Au contraire de Ségolène Royal, que l'évocation de sa voix suffit à ramener à sa condition de femme, le candidat fait preuve d'une puissance vocale qui l'inscrit dans un registre narratif dont le divin n'est pas absent. Les évocations de la voix, nombreuses dans les discours tenus sur Ségolène Royal, sont très rares dans ceux consacrés à Nicolas Sarkozy. Et la seule occurrence du corpus consacrée à la voix du candidat contribue à la construction d'une figure exceptionnelle, qui n'est pas simplement humaine. Dans ces évocations de la voix, comme dans celles de la maternité ou de la beauté, Ségolène Royal apparait comme une femme opposée à un homme dont les capacités le situent au-delà du genre masculin. 


\section{L'émotion : une expression interdite ou une ressource?}

L'émotion est un autre élément important évoqué par Nicole Loraux, car elle est interdite aux déesses du panthéon. Or, l'émotion est décrite par tous les journaux au lendemain du discours de Villepinte et tous sanctionnent de façon positive son irruption dans le processus d'intronisation politique de Ségolène Royal:

Et [Ségolène Royal] d'exprimer ses colères [...] (Libération, d)

C'est à ce moment que l'émotion a semblé étreindre la candidate qui s'est exclamée, yeux brillants et poing serré sur son ventre [...] (Le Monde, $\mathrm{d}$ )

Royal - soulagée - sait [...]

Un peu éprouvée par son discours-fleuve [...]

Le flot verbal se déverse, soudain rapide [...]

Les yeux brillent, la mâchoire est serrée, et la candidate est manifestement emportée par une force qui la dépasse et la galvanise [...] (Aujourd'hui en France, c, d, e)

Royal s'est laissée gagner par l'émotion [...]

Tandis que [...] ses yeux se mouillent [...]

Elle a pourtant su installer avec son public des moments de vraie émotion. (Le Figaro, e)

Les différentes émotions sont donc qualifiées par les journaux comme une véritable ressource politique qui exprime la vérité humaine de la candidate. Comme le remarquent Delphine Dulong et Frédérique Matonti, faisant référence à Pierre Bourdieu, "c'est donc un corps "féminin" que ces femmes [les candidates aux élections municipales de 2001] acceptent de mettre en scène, fragile et pris par les émotions dès qu'il est mis sur la place publique» (Dulong, Matonti, 2005, p. 288). Pourtant, comme le rappelle Nicole Loraux, la déesse Artémis expliquait à Hippolyte qu'il est interdit aux dieux de pleurer sur un mortel. Décrire les émotions de Ségolène Royal, c'est rappeler une qualité fondamentalement humaine et féminine, anthropologiquement incompatible avec la condition politique.

Élément intéressant, les émotions manifestées par Nicolas Sarkozy à Versailles sont tout aussi présentes dans les discours médiatiques:

Dans un discours très travaillé [...] le numéro 2 du gouvernement s'est attaché à se montrer [...] calme et maître de lui-même.

Il a sorti les violons, en se posant comme un homme qui connaît la souffrance [...] Toujours dans le registre de l'émotion, il a fendu l'armure [...] (Libération, c)

Sarkozy, qui a fendu l'armure [...]

Carle ministre de l'intérieur, fendant l'armure [...]

Il était plus personnel qu'à l'accoutumée. (Aujourd'hui en France, a) 
M. Sarkozy, soucieux d'adoucir son image [...]

M. Sarkozy a joué sur plusieurs registres, n'évitant ni la confession intime ni les accents de l'émotion [...]

Le candidat a choisi de se mettre à nu avant d'apparaître en candidat.

(Le Monde, a, b, c)

Il a consacré la moitié de son [discours] à évoquer sa «part d'humanité» [...]

Grâce à l'aveu de ses failles intimes [...]

Souriant et visiblement ému [...] (Le Figaro, a, b, c)

Enfin, la mention des émotions est également présente dans les articles symétriques consacrés à chacun des deux candidats, quoique de façon un peu différente:

Ségolène Royal, heurtée par la «liberté» que s'est offerte François Hollande [...] (Marianne)

Ségolène Royal, qui paraissait nouée par l'anxiété [...] (Le Monde, e).

Sa femme. La femme qu'il a écrit vouloir «aimer toujours» [...] (Marianne)

Et voilà le candidat qui redevient agressif. Dur. Injuste, souvent [...]

Mais, comme la plupart des hommes politiques, il cherche les marques d'une affection jamais assez grande à ses yeux (Le Monde, f).

Alors, quoi? Le candidat serait tombé, en manifestant ses émotions, dans le même piège que celui tendu par la candidate à elle-même par l'évocation de sa féminité? Aussi délicate qu'elle puisse paraitre, la réponse semble pourtant être négative. Il faut remarquer que la figure de Ségolène Royal est affectée par diverses émotions, tandis que Nicolas Sarkozy est décrit comme maitrisant ses émotions, jusques et y compris ses souffrances. Le candidat utilise les émotions comme une ressource politique, ce que Le Monde et L'Express indiquent en rappelant, dans leurs articles symétriques, un "défi » du candidat: "se faire aimer» (Le Monde, f; L'Express, a). Nicolas Sarkozy est candidat, d'ores et déjà inscrit dans le panthéon politique; il exerce sur les autres un pouvoir que cette inscription, non déniée par les discours, lui permet d'exercer. Cela est noté par Libération qui commence le récit du discours de Versailles par la phrase suivante: "On attendait Charlemagne, ce fut pharaon.» (Libération, c) Ce n'est pas un chef qui s'exprime, mais un dieu. Et à ce dieu, protégé de la chute du panthéon parce qu'il est un dieu au masculin, tout semble possible, de l'expression d'une souffrance à celle de ses colères. Comme le précise Nicole Loraux dans son commentaire de Chrysippe, " "on peut même dire que les puissances ont deux sexes - mâles quand elles sont en action, féminines quand elles sont passives de nature". Ainsi, le sexe des dieux relève d'une opération de pensée appariant les puissances et les éléments au masculin ou au féminin » (Loraux, 1991, p. 43). Quoique décrivant les deux candidats sous l'angle des émotions, les représentations médiatiques n'en marquent donc pas moins une différence fondamentale: tremblante, étreinte, heurtée et nouée par l'anxiété, Ségolène Royal subit les 
émotions, est renvoyée à sa condition de femme et rejetée hors du panthéon politique. Nicolas Sarkozy, lui, est acteur de ses émotions; il demeure ainsi qualifié politiquement.

\section{L’oxymore de la proximité présidentielle}

Cet éloignement de Ségolène Royal du panthéon politique est enfin forgé par ce que les journaux montrent du recours de la candidate à la proximité. Car, de même qu'elles ne doivent pas manifester leurs émotions, les déesses doivent garder leurs distances avec les mortels:

C'est une femme en empathie avec les autres [...]

Moquée par l'élite pour incompétence bécassinière, elle ressurgit [...] poussée par la France d'en bas [...]

La socialiste a réussi son pari de donner corps à sa démocratie participative.

(Libération, d, e)

Elle entend faire la différence par sa connaissance sensible, parfois intuitive de la nation.

Très surveillés par une présidente [...] infirmière et institutrice auprès du peuple [...] (L'Express, b)

La candidate socialiste [...] serre des mains, embrasse des joues [...]

«Elle est restée à la fois humble, à l'écoute, et en même temps habitée par le rôle », témoigne sa conseillère spéciale [...]

Dans toutes les enquêtes d'opinion [...] Ségolène Royal est apparue «proche des préoccupations » des Français [...] (Le Monde, e)

En faisant de la proximité le principe fondateur de sa campagne, Ségolène Royal permet la construction de discours qui sanctionnent la qualité qu'elle juge politiquement cardinale, rappelant en cela ce que note Marion Paoletti dans le numéro 77 de la revue Mots. Les langages du politique: «La proximité sociale et la similarité du monde quotidien vécu avec les électeurs se prolongent dans un projet politique et une proximité symbolique.» (Paoletti, 2005, p. 37) Dans ce même numéro, Rémi Lefebvre indique aussi que «la proximité est perçue comme une manière de ressourcer la légitimité politique et de refonder le lien représentatif [...] » (Lefebvre, 2005, p. 41). Les précisions des deux auteurs ne peuvent être discutées pour ce qui est de la campagne pour les élections législatives de 2002 ou du débat sur le cumul des mandats entre 1998 et 2000 . Mais, dans les deux analyses, il est question d'élections relevant de l'échelle infranationale. Or, la campagne pour l'élection présidentielle restreint la sphère politique au panthéon politique. Pour accéder ou appartenir à ce panthéon, la proximité se révèle une ressource piège, car, de même qu'il ne faut pas affronter le regard de la Méduse (le regard qui tue), on ne peut pas regarder le divin en face. Dans l'accession à la présidence de la Répu- 
blique, la proximité relève de l'oxymore. Ségolène Royal fait de la proximité sa ressource clé; ce faisant, elle rompt avec la distance des déesses et permet qu'on la qualifie non plus comme une politique mais comme une femme. Le féminin prime alors sur le politique.

Que dire, alors, de la proximité voulue par Nicolas Sarkozy? Tout d'abord, que son usage de la proximité n'est pas qualifié comme celui de Ségolène Royal, même lorsqu'il apparait dans le discours d'investiture :

Le numéro 2 du gouvernement s'est attaché à se montrer humain [...] en se présentant en "petit Français au sang mêlé ", [...] en se posant comme un homme qui [...] peut comprendre [la souffrance] des autres. (Libération, c)

Il était plus personnel qu'à l'accoutumée. (Aujourd'hui en France, a)

Nicolas Sarkozy veut «tendre la main » à tous les Français.

C'est sur le ton de la confidence que le plus médiatisé des candidats a choisi de s'adresser [...] à «tous les Français».

Grâce à l'aveu de ses failles intimes, Nicolas Sarkozy peut espérer casser l'image qu'il a [...]. Ne plus passer pour le privilégié ami des riches, qui n'a jamais souffert. (Le Figaro, b)

Pour tous les journaux, le candidat fait de la proximité une arme du combat tactique. La sanction narrative est plutôt unanime, qui note l'expression de l'humanité et la volonté d'être proche des électeurs du candidat, mais qui pointe aussi le caractère circonstanciel du changement ainsi affiché. Ensuite, et alors même qu'il n'est pas encore élu, les discours qualifient Nicolas Sarkozy comme capable de préserver la distance qui lui permettra d'exercer pleinement sa fonction :

Un instant, le candidat reste seul [...], le temps de contempler sa mue. (Le Monde, c)

Le 6 mai, s'il l'emporte [...] il partira quelque part, dans un endroit isolé.

"Le temps qu'il me faudra pour habiter la fonction.»

Quant au pouvoir suprême? Il serait «une ascèse, l'oubli de soi et, sans doute, la mise entre parenthèses de son bonheur personnel». (Le Monde, f)

Le candidat de l'UMP est un politique et le signifie en exprimant ce nécessaire oubli de soi et sa distance à la condition simplement humaine. Au contraire de Ségolène Royal, femme-candidate portée par la «France d'en bas» (Libération, b), Nicolas Sarkozy présente une figure plus détachée, que les discours peuvent déjà inscrire dans le panthéon politique.

De façon notable ou plus subtile, les articles retenus tendent à qualifier la figure de Ségolène Royal à partir de son être-femme, lui refusant ainsi l'accès au panthéon politique. Ces tendances convergentes peuvent être nuancées par la présence de très nombreux éléments de discours qui qualifient la candidate comme telle. Ainsi, $L$ 'Express note que la candidate «veut reconstruire la maison 
France en commençant par sa charpente institutionnelle» (L'Express, b). Que son aptitude à la campagne présidentielle soit remarquée ou que ses projets soient détaillés, Ségolène Royal fait l'objet de qualifications qui, tout en notant son être-femme, sanctionnent positivement son être-politique. Ce sont ces éléments de qualification qui apparaissent comme ceux par lesquels les appartenances symboliques pourraient commencer à changer; comme ceux qui tendraient à rendre poreuse la frontière entre le féminin et le politique.

Mais ces nuances ne compensent pas l'ancrage anthropologique des articles analysés. L'exclusion de Ségolène Royal forgée par ces articles est d'autant plus remarquable qu'elle apparait dans des espaces médiatiques qui affichent une égalité de traitement. À cet égard, il faut insister sur les écarts de qualification entre les deux portraits du Monde et entre les deux dossiers de L'Express. Les deux articles de Marianne caricaturent ces écarts et parlent, pour l'un, de Ségolène Royal, pour l'autre, de Cécilia Sarkozy comme femme du candidat dont l'appartenance à son genre ne remet pas en cause celle de Nicolas Sarkozy au panthéon politique. Peut-être faut-il d'ailleurs comprendre les déboires médiatiques de Nicolas Sarkozy, au moment de son mariage avec Carla Bruni, comme relevant d'une soudaine interrogation sur son appartenance au panthéon. En affichant son bonheur d’homme, Nicolas Sarkozy aurait chuté du panthéon et serait redevenu simple mortel. Les discours médiatiques auraient alors exprimé une porosité symbolique entre le masculin et le politique.

Concluons avec Nicole Loraux: "Une déesse: un dieu au féminin? Sans doute. Encore faut-il désormais souligner et le dieu et le féminin comme enjeu de spéculation, comme support de rêveries pour la cité des hommes. " (Loraux, 1991, p. 55) Anthropologiquement ancrée, cette rêverie a continué à interdire aux femmes l'accès aux responsabilités politiques majeures. Il semble cependant que si la campagne présidentielle de 2007 fut un échec pour la candidate, elle aura permis, par la simple présence de celle-ci dans la compétition, de commencer à envisager la présence d'une femme au panthéon.

\section{Références}

ACHIN Catherine, Dorlin Elsa, 2007, “J'ai changé, toi non plus”, La fabrique d’un-e présidentiable: Sarkozy/Royal au prisme du genre», en ligne [URL: http://www. mouvements.info/spip.php?article42], site consulté le 3 mars 2009.

Coulomb-Gully Marlène, 2009, "Le corps présidentiel. Représentation et incarnation dans la campagne présidentielle française de 2007 ", Mots. Les langages du politique, n 89, 2007. Débats pourl'Élysée, p. 25-38.

COURTÉs Joseph, 1991, Analyse sémiotique du discours, de l'énoncé à l'énonciation, Paris, Hachette. 
Dulong Delphine, Matontı Frédérique, 2005, «L'indépassable "féminité". La mise en récit des femmes en campagne», Mobilisations électorales. Le cas des élections municipales de 2001, J. Lagroye, P. Lehingue, F. Sawicki éd., Paris, PUF, p. 281-303.

LefEBVRe Rémi, 2005, «Rapprocher l'élu et le citoyen. La "proximité" dans le débat sur la limitation du cumul des mandats (1998-2000) », Mots. Les langages du politique, $\mathrm{n}^{\circ} 77$, Proximité, p. 41-57.

LoRaux Nicole, 2002 [1991], «Qu'est-ce qu'une déesse?», Histoire des femmes, G. Duby, M. Perrot éd., t. 1, L'Antiquité, P. Schmitt Pantel éd., Paris, Perrin (passage cité: Plon, 1991, p. 39-79).

PAOLETTI Marion, 2005, «Domestiquer la représentation politique. Les professions de foi pour les élections législatives de 2002 ", Mots. Les langages du politiques, $\mathrm{n}^{\circ} 77$, Proximité, p. 29-39.

SouRd Cécile, 2005, "Femmes ou politiques? La représentation des candidates aux élections françaises de 2002 dans la presse hebdomadaire ", Mots. Les langages du politique, $\mathrm{n}^{\circ} 78$, Usages politiques du genre, p. 65-77.

SINGLYFrançois (de), 2007, « "Nicolas et Royal” ou la domination masculine », Le Monde, 16 mars.

\section{Corpus}

L'Humanité

15 janvier 2007

«Sarkozy ajuste son costume de président» (a).

12 février 2007

«Ségolène Royal présente son pacte présidentiel» (b).

«Ne pas noyer le poisson» (c).

\section{Libération}

15 janvier 2007

«Sarkozy adoubé par une droite rassemblée » (a).

«Maître à bord» (b).

« 98,1\% des voix, et lui, et lui, et lui » (c).

12 février 2007

«Ségolène Royal, un pacte plein d'impact» (d).

«L'iconoclaste» (e).

\section{Aujourd'hui en France}

15 janvier 2007

"Sarkozy: “J’ai changé”» (a).

"Cécilia, discrète et omniprésente » (b).

12 février 2007

«Les promesses de Royal remobilisent la gauche» (c).

«Deux heures d'un discours-fleuve» (d).

«"Je sais, en tant que mère"» (e). 
Le Figaro

15 janvier 2007

«Nicolas Sarkozy veut "tendre la main” à tous les Français » (a).

"Le champion de la droite dit "son amour de la France" et affiche sa "part d'humanité"» (b).

12 février 2007

«Le piège de l'ambiguïté» (c).

«Soulagé, le PS attaque la "vraie campagne” » (d).

«Le pacte de Royal joue la gauche et le centre» (e).

Le Monde

16 janvier 2007

«Sarkozy veut rassembler au-delà de son camp» (a).

«Un nouveau Sarkozy» (b).

«M. Sarkozy centre sa campagne sur la France et le travail» (c).

13 février 2007

«Ségolène Royal : cent propositions pour répondre à la crise sociale » (d).

26 avril 2007

«Focus: Les métamorphoses de Ségolène Royal » (e).

27 avril 2007

«Focus: Les métamorphoses de Nicolas Sarkozy» (f).

Marianne

20-27 janvier 2007

«Politique. Les couples infernaux d'hier et d'aujourd'hui ».

L'Express

29 mars-4 avril 2007

«Sarkozy se confie: “Quel président je serai” » (a).

5-11 avril 2007

«Ségolène Royal: “Si je suis élue...”» (b). 